\title{
Stable isotope-resolved metabolomics reveals the abnormal brain glucose catabolism in depression based on chronic unpredictable mild stress rats
}

Ting Ling-hu, ${ }^{\mathrm{a}, \mathrm{b}}$, Shao-bo Liuc, Yao Gao ${ }^{\mathrm{a}, \mathrm{b}}$, Yu-mei Han ${ }^{\mathrm{d}}$, Jun-sheng Tian ${ }^{\mathrm{a}, \mathrm{b} *}$, Xue-mei Qin $^{\mathrm{a}, \mathrm{b} *}$

${ }^{a}$ Modern Research Center for Traditional Chinese Medicine, the Key Laboratory of Chemical Biology and Molecular Engineering of Ministry of Education, Shanxi University, Taiyuan 030006, Shanxi, China

${ }^{b}$ The Institute for Biomedicine and Health, Shanxi University, Taiyuan 030006, Shanxi, China

${ }^{c}$ Key Laboratory of Ethnomedicine of Ministry of Education, Center on Translational Neuroscience, School of Pharmacy, Minzu University of China, Beijing 100081, Beijing, China

${ }^{d}$ School of Physical Education, Shanxi University, Taiyuan 030006, Shanxi, China

*Corresponding Author

Xuemei Qin, E-mail: qinxm@sxu.edu.cn

Junsheng Tian, E-mail: jstian@sxu.edu.cn

Tel/Fax: 0351-7019297

\section{Brief description of the supporting information}

Figure S1 Isotopic distribution of key metabolites at different time points for continuous injection $9.0 \mathrm{~h}$

Figure S2 Total ion chromatogram achieved on T3 column under positive ion mode (a) and negative ion mode (b) 
Figure S3 Comparisons of mass isotopologue distributions (MIDs) of other key metabolites in glycolysis and TCA cycle.

Table S1 Identification information for key metabolites

Table S2 Isotopic distribution information of differential metabolites related to glucose catabolism

\section{Materials and Methods}

\section{Reconstruction of CUMS model}

The control group were housed together, while the model rats were housed individually and randomly exposed to one of stressors as follows: $24 \mathrm{~h}$ of food deprivation; $24 \mathrm{~h}$ of water deprivation; tail clamp for $2 \mathrm{~min}$; noise stimuli for $3 \mathrm{~h}$ by an ultrasonic loudspeaker; swimming in $4^{\circ} \mathrm{C}$ cold water for $5 \mathrm{~min}$; restricted movement for $3 \mathrm{~h}$; foot shocks for 10 times (one shock/2 s,10 s duration); day-night reversal; exposure to an experimental room at $45^{\circ} \mathrm{C}$ for $5 \mathrm{~min}$. One stressor was applied per day and the whole stress procedure lasted for 28 days with a completely random order. Each stimulus factor should not appear continuously and the total number of occurrences should not exceed 4 times. All rats were maintained under standard laboratory conditions $\left(25 \pm 2^{\circ} \mathrm{C}\right.$, $45 \pm 15 \%$ relative humidity, and $12 \mathrm{~h} / 12 \mathrm{~h}$ light/dark cycle), and had free access to distilled water and food except for the period of water and food deprivation.

\section{LC-MS analysis}

Separation of hydrophilic metabolites was achieved on a SeQuant ZIC-cHILIC column (2.1 $\mathrm{mm} \times 150 \mathrm{~mm}, 3 \mu \mathrm{m}$, Millipore Sigma, Burlington, MA, USA) at a constant flow rate of $0.3 \mathrm{~mL} / \mathrm{min}$. The mobile phase $\mathrm{A}$ is ammonium acetate solution $(\mathrm{pH}=3.25 ; 10$ $\mathrm{mM})$ and $\mathrm{B}$ is acetonitrile. The injection volume is $5 \mu \mathrm{L}$. The column temperature is $35^{\circ} \mathrm{C}$. The eluting gradient is $0 \mathrm{~min}, 95 \% \mathrm{~B} ; 0 \sim 8 \mathrm{~min}, 95 \% \sim 85 \% \mathrm{~B} ; 8 \sim 10 \mathrm{~min}, 85 \% \sim 81 \%$ B; 10 22 $\min , 81 \% \sim 60 \% \mathrm{~B}$; 22 25 $\min , 60 \% \sim 95 \% \mathrm{~B} ; 22 \sim 25 \mathrm{~min}, 95 \% \mathrm{~B}$. The ion source adopted HESI and detected in negative ion mode. The mass spectrometry 
parameters are as follows: sheath gas $=55$ arbitrary units, auxiliary gas $=15$ arbitrary units, sweep gas $=3$ arbitrary units, spray voltage $(-)=2.5 \mathrm{kV}$, capillary temperature $=$ $320^{\circ} \mathrm{C}, \mathrm{S}$-lens $\mathrm{RF}$ level $=65.0$, auxiliary gas heater temperature $=450^{\circ} \mathrm{C}$, full scan range $=60 \sim 900(\mathrm{~m} / \mathrm{z})$; resolution $=35,000 ;$ maximum injection time $=120 \mathrm{~ms}$; automatic gain control $(\mathrm{AGC})=1 \mathrm{e}^{6}$ ions.

Separation of hydrophobic metabolites was achieved on a Waters ACQUITY UPLC HSS T3 column $(2.1 \mathrm{~mm} \times 100 \mathrm{~mm}, 1.8 \mu \mathrm{m}$, Waters, Milford, MA, USA $)$ at a constant flow rate of $0.2 \mathrm{~mL} / \mathrm{min}$. The mobile phase $\mathrm{A}$ is water containing $0.1 \%$ formic acid and $\mathrm{B}$ is acetonitrile containing $0.1 \%$ formic acid. The injection volume is $5 \mu \mathrm{L}$. The column temperature is $35^{\circ} \mathrm{C}$. The eluting gradient is $0 \sim 2 \mathrm{~min}, 2 \% \mathrm{~B} ; 2 \sim 3 \mathrm{~min}, 2 \% \sim 35 \% \mathrm{~B}$; 3 28 min, 35\% 98\% B; 28 30 min, 98\% B; 30 32 min, 98\% 2\% B; 32 34 min, 2\% B. The ion source adopted HESI and detected in positive and negative ion switching acquisition mode. The mass spectrometry parameters are as follows: sheath gas $=35$ arbitrary units, auxiliary gas $=10$ arbitrary units, spray voltage $(+)=3.0 \mathrm{kV}$, spray voltage $(-)=2.7 \mathrm{kV}$, capillary temperature $=300^{\circ} \mathrm{C}$, S-lens $\mathrm{RF}$ level $=55.0$, auxiliary gas heater temperature $=300^{\circ} \mathrm{C}$, full scan range $=100 \sim 1500(\mathrm{~m} / \mathrm{z})$; resolution $=35,000$; maximum injection time $=120 \mathrm{~ms}$; automatic gain control $(\mathrm{AGC})=1 \mathrm{e}^{6}$ ions .

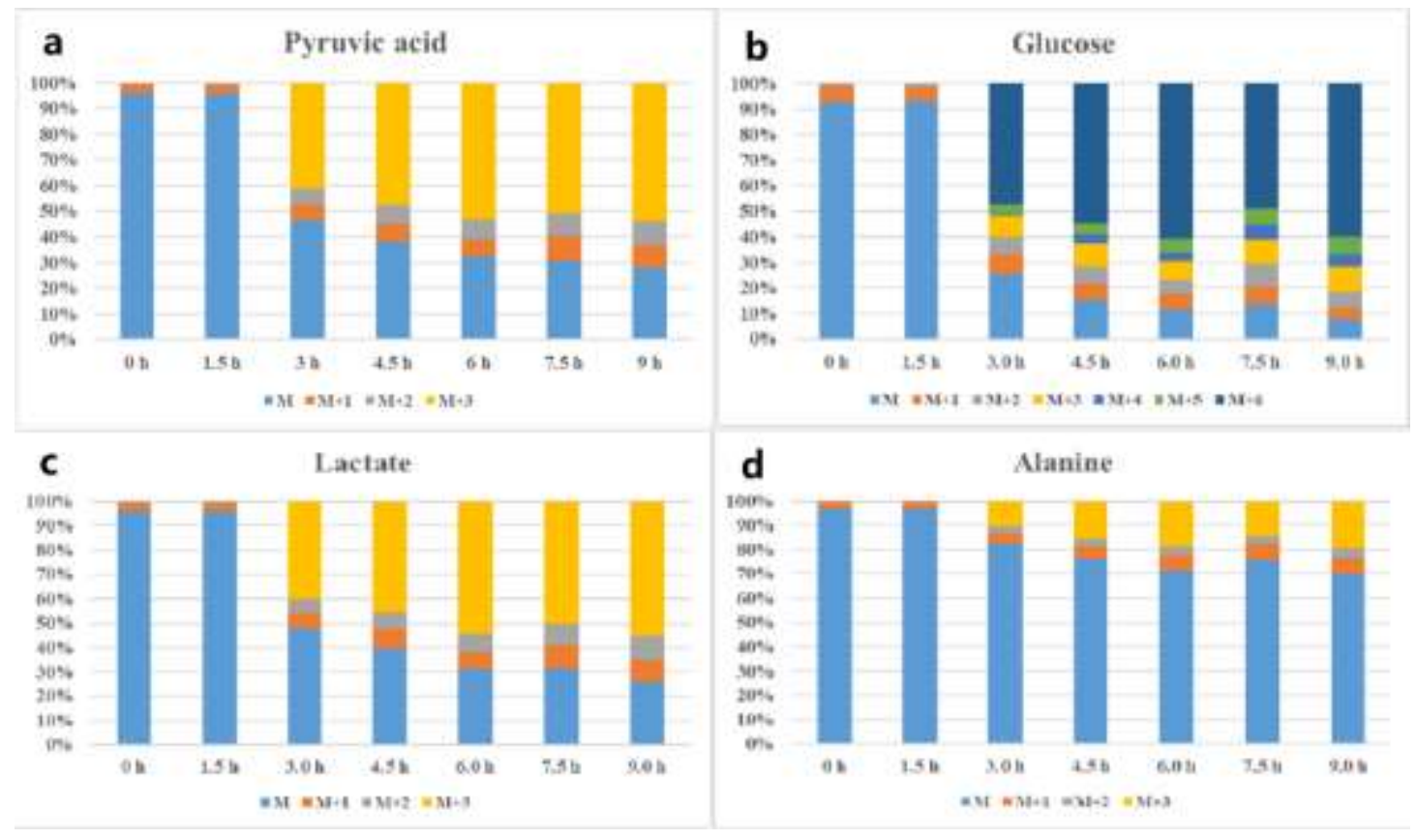


Figure S1 Isotopic distribution of key metabolites at different time points for continuous injection 9.0 h. The isotopic distribution of pyruvic acid(a), glucose (b), lactate (c) and alanine (d).
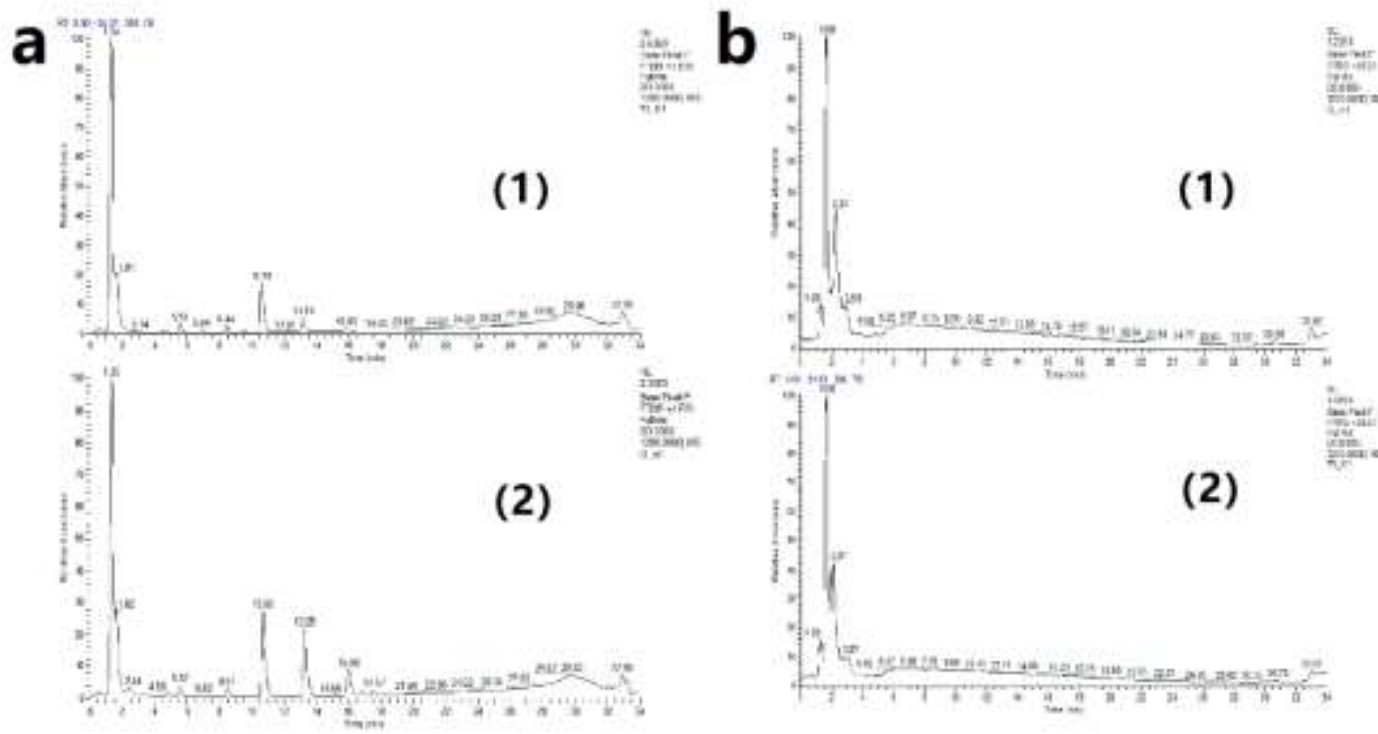

Figure S2 Total ion chromatogram achieved on T3 column under positive ion mode (a) and negative ion mode (b). Upper panel (1) and bottom panel (2) represent control group and CUMS group, respectively.
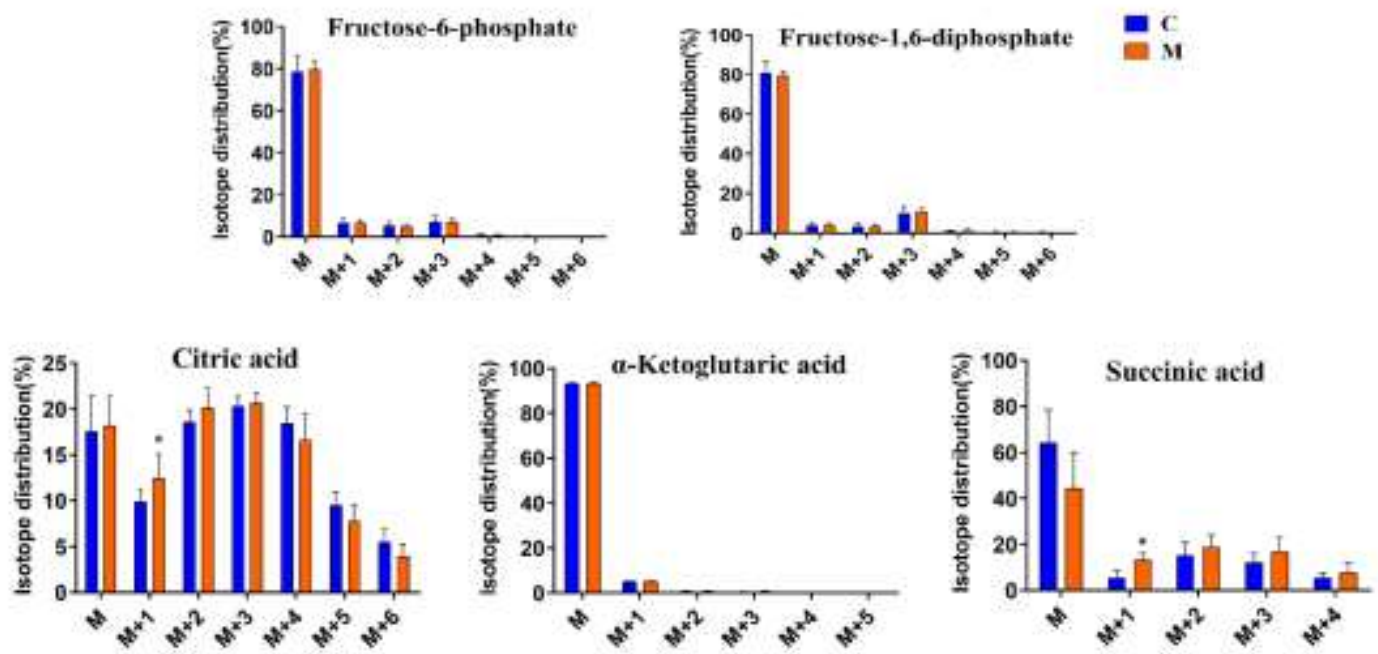

Figure S3 Ccomparisons of mass isotopologue distributions (MIDs) of other key metabolites in glycolysis and TCA cycle. $\mathrm{C}$ and $\mathrm{M}$ represent control group and CUMS 
group $(\mathrm{n}=10)$, respectively. Values given are the means \pm SD. ${ }^{*} p<0.05$ as compared with the control group. 
Table S1 Identification information for key metabolites

\begin{tabular}{|c|c|c|c|c|c|c|c|}
\hline No. & Name & Formula & $\mathrm{RT}(\min )$ & $\mathrm{m} / \mathrm{z}$ & Mode & Column & Fragment ions \\
\hline 1 & Glucose ${ }^{*}$ & C6H12O6 & 15.17 & 179.05611 & $\mathrm{~N}$ & HILIC & $\begin{array}{l}59.01295,71.01297,89.02354,101.02344, \\
119.04958,163.03969\end{array}$ \\
\hline 2 & Fructose-6-phosphate* & C6H13O9P & 20.91 & 259.02271 & $\mathrm{~N}$ & HILIC & $\begin{array}{l}\text { 78.95822, 96.96886, 101.2370, 138.97964, } \\
168.99043,199.00163\end{array}$ \\
\hline 3 & $\begin{array}{l}\text { Fructose-1,6- } \\
\text { diphosphate }^{*}\end{array}$ & C6H14O12P2 & 22.83 & 338.98920 & $\mathrm{~N}$ & HILIC & $\begin{array}{l}\text { 71.01313, 78.95819, 96.96884, 158.92500, } \\
176.93565,241.01215\end{array}$ \\
\hline 4 & Pyruvic acid* & $\mathrm{C} 3 \mathrm{H} 4 \mathrm{O} 3$ & 4.44 & 87.00788 & $\mathrm{~N}$ & HILIC & $52.07136,55.01804,57.03354,85.00339$ \\
\hline 5 & Lactate $^{*}$ & $\mathrm{C} 3 \mathrm{H} 6 \mathrm{O} 3$ & 7.09 & 89.02356 & $\mathrm{~N}$ & HILIC & $\begin{array}{l}53.61600,56.60215,70.08065,71.01284, \\
87.00779\end{array}$ \\
\hline 6 & Alanine* & $\mathrm{C} 3 \mathrm{H} 7 \mathrm{NO} 2$ & 16.5 & 88.03953 & $\mathrm{~N}$ & HILIC & $\begin{array}{l}59.01292,62.00275,71.01316,87.00783 \\
57.03366,67.01805,85.02864,87.00793,\end{array}$ \\
\hline 7 & Citric acid ${ }^{*}$ & $\mathrm{C} 6 \mathrm{H} 8 \mathrm{O} 7$ & 21.87 & 191.01973 & $\mathrm{~N}$ & HILIC & $\begin{array}{l}101.02381, \quad 111.00806, \quad 112.01134, \\
129.01880,147.02953,173.00922\end{array}$ \\
\hline 8 & $\alpha$-Ketoglutaric acid ${ }^{*}$ & $\mathrm{C} 5 \mathrm{H} 6 \mathrm{O} 5$ & 10.56 & 145.01370 & $\mathrm{~N}$ & HILIC & $\begin{array}{l}55.01799,59.01294,71.01294,87.00787 \text {, } \\
99.00811,115.00292\end{array}$ \\
\hline
\end{tabular}


$9 \quad$ Succinic acid*

$10 \quad$ Fumaric acid*

$11 \quad$ Malic acid*

12 Glutamine*

13

Glutamic acid*

$14 \quad$ Aspartic acid*

15 Leucine*

16 Tryptophan*

17 Proline
C4H6O4

8.19

117.0186

C4H4O4

18.63

C4H6O5

18.76

C5H10N2O3

17.31

C5H9O4N

18.73

146.04535

$\mathrm{C} 4 \mathrm{H} 7 \mathrm{NO} 4$

18.76

132.02960

12.35

130.08670

$\mathrm{C} 11 \mathrm{H} 12 \mathrm{~N} 2 \mathrm{O} 2$

5.8

205.09715

$\mathrm{P}$

T3

C5H9NO2
$13.97 \quad 114.0561$
55.01803, 73.02859, 74.03188, 99.00801, 107.55421

54.32011, 70.02891, 71.01298, 72.00819, 89.01366

59.01278, 71.01295, 88.03957, 115.00298, 58.02888, 72.00814, 74.02384, 84.04464, $101.07123,109.04002,127.05064$ 59.01294, 71.01312, 74.02395, 85.02866, $102.05531,128.03470$

$58.02892,71.01295,88.03957,115.00298$ 57.53554, 72.02882, 74.09428, 91.91337, $102.10854,128.03491$ 72.00819, 74.02385, 82.06355, 116.04986, $130.06599, \quad 142.06572, \quad 159.09229$, 186.05569

$55.80551,66.03402,86.02406,112.03971$ 


\begin{tabular}{|c|c|c|c|c|c|c|c|}
\hline 18 & $\begin{array}{l}2, \quad 3, \\
\text { Trihydroxypyridine }\end{array}$ & $\mathrm{C} 5 \mathrm{H} 5 \mathrm{NO} 3$ & 9.02 & 126.0197 & $\mathrm{~N}$ & HILIC & $\begin{array}{l}55.01803,66.03395,82.02898,83.03235 \\
96.99239,110.02419,124.00677\end{array}$ \\
\hline 19 & 5, 5-Dimethylhydantoin & $\mathrm{C} 5 \mathrm{H} 8 \mathrm{~N} 2 \mathrm{O} 2$ & 1.21 & 129.0659 & $\mathrm{P}$ & $\mathrm{T} 3$ & $\begin{array}{l}56.05009,81.04508,83.06078,85.04800, \\
100.03958,102.05501,116.10079\end{array}$ \\
\hline 20 & 4-Oxoproline & $\mathrm{C} 5 \mathrm{H} 7 \mathrm{NO} 3$ & 17.32 & 128.0353 & $\mathrm{~N}$ & HILIC & $\begin{array}{l}67.02926,71.02406,82.02889,84.04462, \\
99.05560,107.02450,109.04005\end{array}$ \\
\hline 21 & 2-Oxoglutaramate & $\mathrm{C} 5 \mathrm{H} 7 \mathrm{NO} 4$ & 9.01 & 144.0302 & $\mathrm{~N}$ & HILIC & $\begin{array}{l}59.01296,71.01294,72.00822,87.00800, \\
99.01931,101.06009,115.00336\end{array}$ \\
\hline 22 & O-Phospho-L-serine & $\mathrm{C} 3 \mathrm{H} 8 \mathrm{NO} 6 \mathrm{P}$ & 18.71 & 184.0017 & $\mathrm{~N}$ & HILIC & $\begin{array}{ll}72.00832, \quad 100.03960, & 128.03441, \\
150.08829, & 165.99088\end{array}$ \\
\hline 23 & Inosine & $\mathrm{C} 10 \mathrm{H} 12 \mathrm{~N} 4 \mathrm{O} 5$ & 10.34 & 267.0735 & $\mathrm{~N}$ & HILIC & $\begin{array}{l}65.01378, \quad 92.02455, \quad 108.01958, \\
126.03028,149.04663,177.04118\end{array}$ \\
\hline 24 & N-Acetylneuraminic acid & C11H19NO9 & 17.17 & 308.0987 & $\mathrm{~N}$ & HILIC & $\begin{array}{l}59.01298,71.04928,87.00793,98.06031, \\
119.03412,124.00680,170.04555\end{array}$ \\
\hline
\end{tabular}

* represents compounds identified by standards. 
Table S2 Isotopic distribution information of differential metabolites related to glucose catabolism

\begin{tabular}{|c|c|c|c|c|c|}
\hline Name & Isomers & $\mathrm{C}(\%)$ & $\mathrm{M}(\%)$ & $p$ & $\mathrm{FC}$ \\
\hline \multirow[t]{4}{*}{ Pyruvic acid } & M & $80.451 \pm 5.16$ & $84.733 \pm 4.997$ & $0.016^{*}$ & 1.05 \\
\hline & $\mathrm{M}+1$ & $3.358 \pm 1.688$ & $3.332 \pm 1.793$ & 0.964 & 0.99 \\
\hline & $\mathrm{M}+2$ & $3.395 \pm 1.135$ & $2.63 \pm 1.396$ & 0.070 & 0.77 \\
\hline & $\mathrm{M}+3$ & $12.796 \pm 3.222$ & $9.305 \pm 2.691$ & $0.002^{\#}$ & 0.73 \\
\hline \multirow[t]{4}{*}{ Lactate } & M & $54.174 \pm 4.627$ & $59.035 \pm 6.44$ & $0.000^{\#}$ & 1.09 \\
\hline & $\mathrm{M}+1$ & $5.173 \pm 2.212$ & $5.534 \pm 1.947$ & 0.490 & 1.07 \\
\hline & $\mathrm{M}+2$ & $6.007 \pm 1.213$ & $5.648 \pm 1.789$ & 0.257 & 0.94 \\
\hline & $\mathrm{M}+3$ & $34.646 \pm 3.882$ & $29.783 \pm 5.875$ & $0.000^{\#}$ & 0.86 \\
\hline \multirow[t]{4}{*}{ Alanine } & M & $67.332 \pm 4.64$ & $71.723 \pm 5.742$ & 0.095 & 1.07 \\
\hline & $\mathrm{M}+1$ & $19.805 \pm 4.108$ & $13.441 \pm 3.006$ & $0.005^{\#}$ & 0.68 \\
\hline & $M+2$ & $2.719 \pm 1.474$ & $2.831 \pm 1.257$ & 0.871 & 1.04 \\
\hline & $\mathrm{M}+3$ & $10.145 \pm 4.583$ & $12.006 \pm 3.362$ & 0.375 & 1.18 \\
\hline \multirow[t]{4}{*}{ O-Phospho-L- } & M & $19.195 \pm 0.958$ & $20.448 \pm 4.058$ & 0.367 & 1.07 \\
\hline & $\mathrm{M}+1$ & $13.184 \pm 1.614$ & $17.999 \pm 5.038$ & $0.016^{*}$ & 1.37 \\
\hline & $M+2$ & $31.209 \pm 1.197$ & $30.232 \pm 6.581$ & 0.655 & 0.97 \\
\hline & $M+3$ & $36.411 \pm 2.831$ & $31.322 \pm 9.527$ & 0.137 & 0.86 \\
\hline \multirow[t]{5}{*}{ Fumaric acid } & M & $42.482 \pm 1.593$ & $43 \pm 9.689$ & 0.893 & 1.01 \\
\hline & $\mathrm{M}+1$ & $12.514 \pm 2.069$ & $17.134 \pm 3.816$ & $0.020 *$ & 1.37 \\
\hline & $\mathrm{M}+2$ & $16.963 \pm 0.911$ & $18.619 \pm 2.099$ & 0.091 & 1.10 \\
\hline & $\mathrm{M}+3$ & $16.882 \pm 1.142$ & $14.557 \pm 3.635$ & 0.149 & 0.86 \\
\hline & $\mathrm{M}+4$ & $11.158 \pm 2.227$ & $6.689 \pm 2.615$ & $0.005^{\#}$ & 0.60 \\
\hline \multirow[t]{4}{*}{ Malic acid } & M & $12.965 \pm 1.974$ & $21.137 \pm 5.071$ & $0.002^{\#}$ & 1.63 \\
\hline & $\mathrm{M}+1$ & $16.906 \pm 2.414$ & $21.839 \pm 4.114$ & $0.020 *$ & 1.29 \\
\hline & $\mathrm{M}+2$ & $26.536 \pm 1.678$ & $24.256 \pm 2.199$ & 0.060 & 0.91 \\
\hline & $\mathrm{M}+3$ & $24.918 \pm 3.157$ & $22.015 \pm 6.662$ & 0.314 & 0.88 \\
\hline
\end{tabular}




\begin{tabular}{|c|c|c|c|c|}
\hline & $M+4$ & $18.675 \pm 3.733$ & $10.754 \pm 3.772$ & $0.005^{\#}$ \\
\hline \multirow[t]{5}{*}{ Aspartic acid } & $\mathrm{M}$ & $45.141 \pm 5.226$ & $42.718 \pm 9.236$ & 0.444 \\
\hline & $\mathrm{M}+1$ & $10.747 \pm 1.998$ & $14.857 \pm 2.568$ & $0.000^{\#}$ \\
\hline & $\mathrm{M}+2$ & $16.024 \pm 1.715$ & $18.438 \pm 3.813$ & 0.065 \\
\hline & $\mathrm{M}+3$ & $16.5 \pm 1.436$ & $14.708 \pm 3.047$ & 0.086 \\
\hline & $M+4$ & $11.589 \pm 2.373$ & $9.279 \pm 4.77$ & 0.156 \\
\hline \multirow[t]{5}{*}{ Asparagine } & $\mathrm{M}$ & $69.129 \pm 6.38$ & $63.731 \pm 8.362$ & 0.119 \\
\hline & $\mathrm{M}+1$ & $11.238 \pm 4.014$ & $12.253 \pm 2.184$ & 0.509 \\
\hline & $\mathrm{M}+2$ & $10.525 \pm 2.103$ & $11.885 \pm 3.039$ & 0.254 \\
\hline & $\mathrm{M}+3$ & $5.29 \pm 0.524$ & $7.288 \pm 2.784$ & $0.040 *$ \\
\hline & $M+4$ & $3.818 \pm 0.858$ & $4.843 \pm 3.154$ & 0.323 \\
\hline \multirow[t]{6}{*}{ Proline } & $\mathrm{M}$ & $94.429 \pm 2$ & $93.675 \pm 2.752$ & 0.516 \\
\hline & $\mathrm{M}+1$ & $0.664 \pm 0.344$ & $1.108 \pm 0.408$ & $0.024 *$ \\
\hline & $\mathrm{M}+2$ & $1.148 \pm 0.539$ & $1.572 \pm 0.799$ & 0.209 \\
\hline & $\mathrm{M}+3$ & $1.575 \pm 0.451$ & $1.725 \pm 0.794$ & 0.631 \\
\hline & $M+4$ & $1.24 \pm 0.449$ & $1.196 \pm 0.669$ & 0.871 \\
\hline & $\mathrm{M}+5$ & $0.944 \pm 0.47$ & $0.725 \pm 0.589$ & 0.396 \\
\hline
\end{tabular}

$2, \quad 3, \quad 6-$

Trihydroxypyridine

$\begin{array}{lllll}\text { M } & 34.955 \pm 6.732 & 28.177 \pm 6.338 & 0.089 & 0.81\end{array}$

$\begin{array}{lllll}M+1 & 8.089 \pm 1.861 & 10.791 \pm 1.266 & 0.011^{*} & 1.33 \\ \mathrm{M}+2 & 22.406 \pm 2.268 & 23.375 \pm 4.092 & 0.620 & 1.04 \\ \mathrm{M}+3 & 12.115 \pm 3.843 & 18.147 \pm 4.112 & 0.021^{*} & 1.50 \\ \mathrm{M}+4 & 13.542 \pm 2.261 & 12.143 \pm 2.569 & 0.325 & 0.90 \\ \mathrm{M}+5 & 8.893 \pm 1.008 & 7.367 \pm 1.451 & 0.059 & 0.83\end{array}$

$5, \quad 5$

Dimethylhydantoin

$\begin{array}{lllll}\text { M } & 21.085 \pm 3.206 & 23.28 \pm 5.16 & 0.226 & 1.10\end{array}$

$\begin{array}{lllll}\mathrm{M}+1 & 11.065 \pm 1.804 & 13.446 \pm 2.202 & 0.009^{\#} & 1.22 \\ \mathrm{M}+2 & 21.185 \pm 2.071 & 22.678 \pm 1.772 & 0.071 & 1.07 \\ & 22.123 \pm 1.97 & & & \\ \mathrm{M}+3 & & 21.346 \pm 3.71 & 0.530 & 0.96\end{array}$




\begin{tabular}{|c|c|c|c|c|c|}
\hline & $\mathrm{M}+4$ & $14.391 \pm 2.167$ & $12.06 \pm 3.629$ & 0.072 & 0.84 \\
\hline & $M+5$ & $10.152 \pm 2.359$ & $7.19 \pm 2.9$ & $0.012 *$ & 0.71 \\
\hline \multirow[t]{6}{*}{ 2-Oxoglutaramate } & M & $34.071 \pm 8.119$ & $32.468 \pm 6.138$ & 0.608 & 0.95 \\
\hline & $\mathrm{M}+1$ & $7.193 \pm 2.596$ & $11.541 \pm 4.482$ & $0.013^{*}$ & 1.60 \\
\hline & $\mathrm{M}+2$ & $19.022 \pm 3.277$ & $20.116 \pm 4.765$ & 0.538 & 1.06 \\
\hline & $\mathrm{M}+3$ & $16.205 \pm 3.102$ & $17.675 \pm 5.005$ & 0.419 & 1.09 \\
\hline & $\mathrm{M}+4$ & $11.013 \pm 2.675$ & $8.654 \pm 2.978$ & 0.065 & 0.79 \\
\hline & $M+5$ & $12.496 \pm 5.656$ & $9.546 \pm 4.678$ & 0.198 & 0.76 \\
\hline \multirow[t]{6}{*}{ Glutamic acid } & M & $12.682 \pm 1.587$ & $16.636 \pm 3.522$ & $0.010 *$ & 1.31 \\
\hline & $\mathrm{M}+1$ & $9.435 \pm 1.712$ & $13.446 \pm 3.587$ & $0.011 *$ & 1.43 \\
\hline & $M+2$ & $20.517 \pm 2.002$ & $24.203 \pm 1.796$ & $0.000^{\#}$ & 1.18 \\
\hline & $\mathrm{M}+3$ & $24.106 \pm 0.688$ & $22.188 \pm 1.784$ & $0.012 *$ & 0.92 \\
\hline & $M+4$ & $18.826 \pm 1.664$ & $14.85 \pm 3.748$ & $0.014^{*}$ & 0.79 \\
\hline & $\mathrm{M}+5$ & $14.434 \pm 3.324$ & $8.677 \pm 3.259$ & $0.001^{\#}$ & 0.60 \\
\hline \multirow[t]{6}{*}{ Glutamine } & M & $18.644 \pm 2.103$ & $23.639 \pm 4.693$ & $0.009^{\#}$ & 1.27 \\
\hline & $\mathrm{M}+1$ & $10.06 \pm 1.807$ & $13.052 \pm 2.35$ & $0.004^{\#}$ & 1.30 \\
\hline & $\mathrm{M}+2$ & $22.219 \pm 3.202$ & $22.074 \pm 4.656$ & 0.935 & 0.99 \\
\hline & $\mathrm{M}+3$ & $21.594 \pm 1.25$ & $19.838 \pm 2.414$ & 0.058 & 0.92 \\
\hline & $\mathrm{M}+4$ & $16.072 \pm 1.954$ & $13.202 \pm 3.882$ & 0.054 & 0.82 \\
\hline & $M+5$ & $11.41 \pm 2.648$ & $8.195 \pm 3.135$ & $0.019 *$ & 0.72 \\
\hline \multirow[t]{9}{*}{ Inosine } & M & $91.281 \pm 1.652$ & $90.636 \pm 1.046$ & 0.358 & 0.99 \\
\hline & $M+1$ & $0.896 \pm 0.503$ & $1.298 \pm 0.556$ & 0.160 & 1.45 \\
\hline & $\mathrm{M}+2$ & $1.867 \pm 0.605$ & $1.955 \pm 0.19$ & 0.687 & 1.05 \\
\hline & $M+3$ & $1.694 \pm 0.217$ & $1.956 \pm 0.166$ & $0.016^{*}$ & 1.15 \\
\hline & $\mathrm{M}+4$ & $1.204 \pm 0.196$ & $1.379 \pm 0.59$ & 0.474 & 1.15 \\
\hline & $M+5$ & $3.025 \pm 0.872$ & $2.743 \pm 0.412$ & 0.410 & 0.91 \\
\hline & $\mathrm{M}+6$ & $0.028 \pm 0.04$ & $0.025 \pm 0.02$ & 0.844 & 0.89 \\
\hline & $\mathrm{M}+7$ & $0.005 \pm 0.008$ & $0.006 \pm 0.009$ & 0.789 & 1.25 \\
\hline & $\mathrm{M}+8$ & - & - & - & - \\
\hline
\end{tabular}


M+9

$\mathrm{M}+10$

N-

$87.738 \pm 2.622$

$82.306 \pm 5.156$

$0.016 *$

0.94

acid

$\begin{array}{lllll}\mathrm{M}+1 & 0.356 \pm 0.296 & 0.468 \pm 0.262 & 0.424 & 1.31 \\ \mathrm{M}+2 & 0.475 \pm 0.14 & 0.613 \pm 0.274 & 0.210 & 1.29 \\ \mathrm{M}+3 & 4.114 \pm 0.938 & 4.268 \pm 0.889 & 0.734 & 1.04 \\ \mathrm{M}+4 & 0.001 \pm 0.001 & 0 \pm 0 & 0.206 & 0.00 \\ \mathrm{M}+5 & 0.181 \pm 0.099 & 0.68 \pm 0.475 & 0.014^{*} & 3.75 \\ \mathrm{M}+6 & 0.28 \pm 0.165 & 0.99 \pm 0.582 & 0.006^{\#} & 3.54 \\ \mathrm{M}+7 & 0.924 \pm 0.241 & 2.192 \pm 1.711 & 0.058 & 2.37 \\ \mathrm{M}+8 & 1.174 \pm 0.374 & 2.107 \pm 0.87 & 0.014^{*} & 1.79 \\ \mathrm{M}+9 & 1.658 \pm 0.457 & 2.512 \pm 0.981 & 0.038^{*} & 1.52 \\ \mathrm{M}+10 & 1.656 \pm 0.393 & 2.229 \pm 0.894 & 0.109 & 1.35 \\ \mathrm{M}+11 & 1.442 \pm 0.389 & 1.635 \pm 0.701 & 0.490 & 1.13\end{array}$

${ }^{*} p<0.05$ and $^{\#} p<0.01$ as compared with the control group; Fold Change $>1.20$ or Fold Change $<0.83$ were set as the thresholds for significantly differential metabolites. 